\title{
An efficient one pot synthesis of some new 1, 4-dihydropyridine derivatives using multicomponent reaction
}

\author{
Devang R. Prajapati, Hardik K. Senjani and Yogesh T. Naliapara* \\ Department of Chemistry, Saurashtra University, Rajkot, Gujarat (India) \\ *Email address: naliaparachem@yahoo.com
}

Keywords: 1, 4-dihydropyridine, One-pot synthesis, multicomponent reactions.

\begin{abstract}
Synthesize target molecules, multicomponent reaction of 4-(4aminophenyl)morpholin-3-one with various pyrazole aldehydes and ethylacetoacetate or methylacetoacetate afforded various 1,4-dihydropyridines derivatives. The newly synthesized compounds were characterized by IR, Mass, ${ }^{1} \mathrm{H}$ NMR, ${ }^{13} \mathrm{C}$ NMR spectroscopy and elemental analysis.
\end{abstract}

\section{INTRODUCTION}

The biological importance of 1,4-dihydropyridines is well documented. Various substituted derivatives of these heterocycles have shown utility against a range of biological targets. A large number of DHP and related compounds have been reported for their anti-inflammatory activity[14].Some new work is also reported so far on the anti-inflammatory activity of 1,4-dihydropyridines possessing analgesic,[5] hypotensive',[6] anti-tumor,[7] and coronary dialating activities[8].

Various methodologies have been described for the synthesis of 1,4-dihydropyridines. During the course of our ongoing interest on synthesis of various heterocyclic compounds using active methylene group containing compounds, we observed that all these compounds are versatile intermediate for the synthesis of dihydropyridines.

\section{EXPERIMENTAL SECTION}

Thin-layer chromatography was accomplished on 0.2-mm precoated plates of silica gel G60 F254 (Merck). Visualization was made with UV light (254 and 365nm) or with an iodine vapor. IR spectra were recorded on a FTIR-8400 spectrophotometer using DRS prob. ${ }^{1} \mathrm{H}(400 \mathrm{MHz}),{ }^{13} \mathrm{C}(100$ $\mathrm{MHz}$ ) NMR spectra were recorded on a Bruker AVANCE II spectrometer in $\mathrm{CDCl}_{3}$ and DMSO. Chemical shifts are expressed in $\delta$ ppm downfield from TMS as an internal standard. Mass spectra were determined using direct inlet probe on a GCMS-QP 2010 mass spectrometer (Shimadzu). Solvents were evaporated with a BUCHI rotary evaporator. Melting points were measured in open capillaries and are uncorrected.

\subsection{General synthesis of (E)-1-phenyl-2-(1-phenylethylidene) hydrazine(1) :-}

Appropriately substituted Acetophenone $(0.1$ mole) was dissolved in $50 \mathrm{ml}$ of ethanol into $250 \mathrm{ml}$ beaker. Phenyl hydrazine $(0.1$ mole) was added to above flask along with 3-4 drops of glacial acetic acid. The reaction mixture was stirred for 1 hour at room temperature. The progress and the completion of reaction were checked by thin layer chromatography using ethyl acetate : hexane $(6: 4)$ as a mobile phase. After the completion of the reaction, the reaction mixture was kept to room temperature for 1 hours and the crystalline product was separated by filtration. The product was washed with ethanol and dried to give substituted acetone phenyl hydrazone in good yield which was pure enough to use as such for the next step. 


\subsection{General synthesis of 1-(1,3-diphenyl-1H-pyrazol-4-yl)ethanone(2) :-}

Dimethylformamide ( 0.32 mole) was transferred into $25 \mathrm{ml}$ flat bottom flak. Phosphorous oxychloride $\left(0.032\right.$ mole) was added drop wise to above flask under stirring at $0-5^{\circ} \mathrm{C}$. After completion of the addition, the mixture was stirred at this temperature for 10-15 min. freshly prepared acetophenonehydrazone 0.03 mole was added to above mixture and the content was heated on water bath for 5-6 hours. The progress and the completion of reaction were checked by thin layer chromatography. After the reaction to be completed, the reaction mixture was cooled to room temperature and the content of the flask was poured on crushed ice to isolate the product. The separated product was filtered off and it was washed with cold water to remove acidity. It was dried at $65^{\circ} \mathrm{C}$ and recrystallized from the mixture of DMF-Methanol to give crystalline pyrazole aldehyde in good yield.

\subsection{General synthesis of various substituted 1,4-dihydropyridines (3A-3T) :-}

A mixture of pyrazole aldehyde $(0.01 \mathrm{~mol})$, ethylacetoacetate or methylacetoacetae $(0.02 \mathrm{~mol})$ in ethanol with few drops of piperidine as base was stirred for $1 \mathrm{hrs}$ at $65-70^{\circ} \mathrm{C}$ in a stoppered flask. After formation of arylidine compound (checked by TLC), 4-(4-aminophenyl)morpholin-3-one was added to the reaction mixture. The reaction mixture was refluxed for 18-20hours. During the reaction the progress and the completion of reaction were checked by thin layer chromatography. After the reaction to be completed, the reaction mixture was kept to room temperature for 1 hours and the reaction mixture was poured into crushed ice.The solid product was separated by filtration. The solid crude product was washed with ethanol, crystallized from methanol.

\section{REACTION SCHEME}
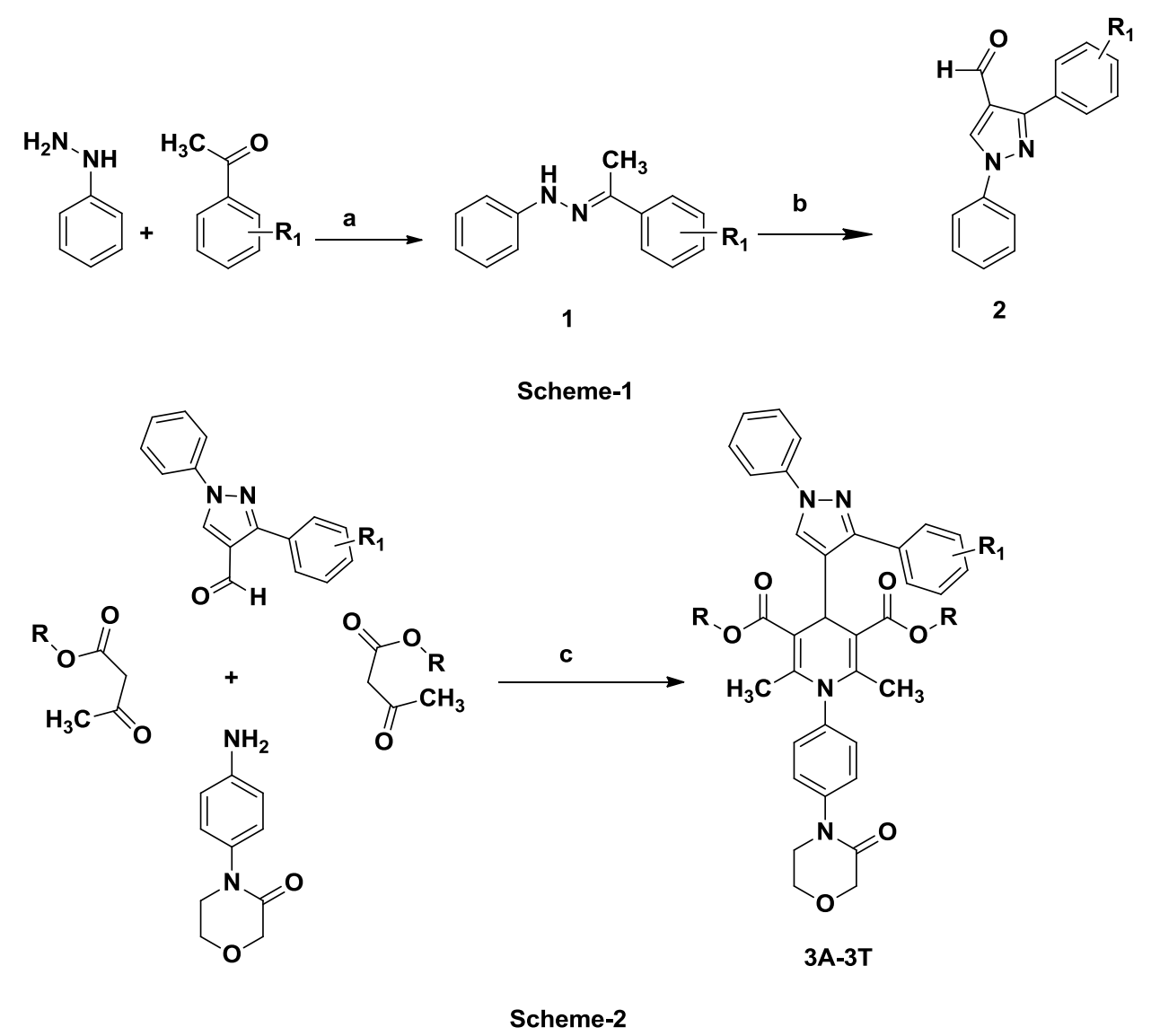

Scheme 1. (a) glacial acetic acid, ethanol, reflux for 5-6 hours; (b) DMF-POCl $3,70-80^{\circ} \mathrm{C}$, 5-6 hours;

Scheme 2. (c) 1-2 drops conc.HCl, ethanol, reflux. 
Table 1. Physical constant of 1,4-dihydropyridines derivatives (3A-3T)

\begin{tabular}{|c|c|c|c|c|c|c|}
\hline Sr.no & Comp. & $\mathbf{R}$ & $\mathbf{R}_{\mathbf{1}}$ & Time hr. & Yield \% & Melting Range $^{\circ} \mathrm{C}$ \\
\hline 1 & $3 \mathrm{~A}$ & $-\mathrm{C}_{2} \mathrm{H}_{5}$ & $\mathrm{H}$ & 20 & 84 & $238-241$ \\
\hline 2 & $3 \mathrm{~B}$ & $-\mathrm{C}_{2} \mathrm{H}_{5}$ & $4-\mathrm{CH}_{3}$ & 18 & 86 & $250-252$ \\
\hline 3 & $3 \mathrm{C}$ & $-\mathrm{C}_{2} \mathrm{H}_{5}$ & $4-\mathrm{F}$ & 22 & 80 & $264-266$ \\
\hline 4 & $3 \mathrm{D}$ & $-\mathrm{C}_{2} \mathrm{H}_{5}$ & $4-\mathrm{Cl}$ & 21 & 78 & $236-238$ \\
\hline 5 & $3 \mathrm{E}$ & $-\mathrm{C}_{2} \mathrm{H}_{5}$ & $4-\mathrm{Br}$ & 23 & 80 & $238-240$ \\
\hline 6 & $3 \mathrm{~F}$ & $-\mathrm{C}_{2} \mathrm{H}_{5}$ & $3-\mathrm{Br}$ & 23 & 82 & $236-240$ \\
\hline 7 & $3 \mathrm{G}$ & $-\mathrm{C}_{2} \mathrm{H}_{5}$ & $2-\mathrm{OCH}_{3}$ & 24 & 75 & $220-225$ \\
\hline 8 & $3 \mathrm{H}$ & $-\mathrm{C}_{2} \mathrm{H}_{5}$ & $4-\mathrm{OCH}_{3}$ & 24 & 78 & $226-230$ \\
\hline 9 & $3 \mathrm{I}$ & $-\mathrm{C}_{2} \mathrm{H}_{5}$ & $3-\mathrm{NO}_{2}$ & 26 & 69 & $250-252$ \\
\hline 10 & $3 \mathrm{~J}$ & $-\mathrm{C}_{2} \mathrm{H}_{5}$ & $4-\mathrm{NO}_{2}$ & 25 & 71 & $250-255$ \\
\hline 11 & $3 \mathrm{~K}$ & $-\mathrm{CH}_{3}$ & $\mathrm{H}$ & 18 & 86 & $252-255$ \\
\hline 12 & $3 \mathrm{~L}$ & $-\mathrm{CH}_{3}$ & $4-\mathrm{CH}_{3}$ & 20 & 87 & $236-238$ \\
\hline 14 & $3 \mathrm{M}$ & $-\mathrm{CH}_{3}$ & $4-\mathrm{F}$ & 23 & 76 & $242-245$ \\
\hline 15 & $3 \mathrm{~N}$ & $-\mathrm{CH}_{3}$ & $4-\mathrm{Cl}$ & 24 & 73 & $255-258$ \\
\hline 16 & 30 & $-\mathrm{CH}_{3}$ & $4-\mathrm{Br}$ & 24 & 71 & $263-266$ \\
\hline 17 & $3 \mathrm{P}$ & $-\mathrm{CH}_{3}$ & $3-\mathrm{Br}$ & 23 & 75 & $250-254$ \\
\hline 18 & $3 \mathrm{Q}$ & $-\mathrm{CH}_{3}$ & $2-\mathrm{OCH}_{3}$ & 22 & 80 & $266-268$ \\
\hline 19 & $3 R$ & $-\mathrm{CH}_{3}$ & $4-\mathrm{OCH}_{3}$ & 21 & 82 & $260-265$ \\
\hline 20 & $3 \mathrm{~S}$ & $-\mathrm{CH}_{3}$ & $3-\mathrm{NO}_{2}$ & 24 & 70 & $244-246$ \\
\hline 21 & $3 \mathrm{~T}$ & $-\mathrm{CH}_{3}$ & $4-\mathrm{NO}_{2}$ & 25 & 73 & $234-238$ \\
\hline
\end{tabular}

\section{SPECTRAL DATA}

Diethyl-4-(1,3-diphenyl-1H-pyrazol-4-yl)-2,6-dimethyl-1-(4-(3-oxomorpholino)phenyl)-1,4dihydropyridine-3,5-dicarboxylate(3A): Brown solid; melting range $238-241^{\circ} \mathrm{C}$; IR (KBr): 3742 , 3377, 3095, 2974, 2324, 2130, 1919, 1735, 1550, 1236, 1192, 1001, 923, 833, 783, 725, 636, 588, 505, $443 \mathrm{~cm}^{-1}$; ${ }^{\mathrm{H}}$ NMR: $\delta 1.100-1.140(\mathrm{t}, 6 \mathrm{H}, J=6.9 \mathrm{~Hz}), 2.239-2.241(\mathrm{~s}, 6 \mathrm{H}), 3.466-3.490(\mathrm{t}, 2 \mathrm{H})$, 3.510-3.534 (t, 2H), 3.936-4.064 (q, 2H), $4.310(\mathrm{~s}, 2 \mathrm{H}) 4.486-4.637(\mathrm{q}, 2 \mathrm{H}), 5.447-5.457(\mathrm{~s}, 1 \mathrm{H})$, 6.783-7.333 (d, 4H, $J=6.8 \mathrm{~Hz}), 7.394-7.419(\mathrm{t}, 2 \mathrm{H}), 7.420-7.467(\mathrm{t}, 1 \mathrm{H}), 7.468-7.488(\mathrm{t}, 1 \mathrm{H}$,$) ,$ $7.490-7.531(\mathrm{t}, 2 \mathrm{H}), 7.664-7.692(\mathrm{~d}, 2 \mathrm{H}, J=6.5 \mathrm{~Hz}), 7.697-7.733(\mathrm{~d}, 2 \mathrm{H}), 8.607(\mathrm{~s}, 1 \mathrm{H}) ;{ }^{13} \mathrm{C}$ NMR: $14.17,16.30,27.17,41.79,60.06,64.31,66.67,104.30,118.45,122.49,123.00,127.55,128.47$, 129.39, 129.59, 130.66, 137.81, 140.05, 141.19, 158.44, 165.55, 167.72; MS (m/z): $647\left(\mathrm{M}^{+}\right)$; Anal. Calcd forC ${ }_{38} \mathrm{H}_{38} \mathrm{~N}_{4} \mathrm{O}_{6}$ : C, 70.57; H, 5.92; N, 8.66; Found: C, 70.60; H, 5.90; N, 8.76.

Diethyl 2,6-dimethyl-1-(4-(3-oxomorpholino)phenyl)-4-(1-phenyl-3-(p-tolyl)-1 H-pyrazol-4-yl)1,4-dihydropyridine-3,5-dicarboxylate(3B): Brownsolid; melting range $250-252^{\circ} \mathrm{C}$; IR (KBr):3738, 3383, 2324, 1919, 1730, 1550, 1236, 1170, 1072, 999, 922, 895, 792, 767, 684, 646, $559,455 \mathrm{~cm}^{-1}$; ${ }^{1} \mathrm{H}$ NMR: $\delta 1.100-1.145(\mathrm{t}, 6 \mathrm{H}, J=6.7 \mathrm{~Hz}), 2.240-2.242(\mathrm{~s}, 6 \mathrm{H}), 2.328-2.333(\mathrm{~s}, 3 \mathrm{H})$, 3.466-3.490 (t, 2H), 3.513-3.536 (t, 2H), 4.230-4.299 (q, 2H), $4.315(\mathrm{~s}, 2 \mathrm{H}), 4.334-4.470(\mathrm{q}, 2 \mathrm{H})$, 5.449-5.460 (s, 1H), 6.774-6.804 (d, 2H, J=6.5Hz), 7.187-7.217 (d, 2H), 7.269-7.299 (d, 2H), 7.395-7.414 (d, 2H), 7.419-7.445 (t, 2H), 7.498-7.546 (t, 1H), 7.664-7.702 (d, 2H), $8.612(\mathrm{~s}$, 
$1 \mathrm{H}){ }^{13} \mathrm{C}$ NMR: $13.90,16.35,27.20,41.81,60.09,64.35,66.70,104.35,118.48,122.48,123.12$, $127.58,128.50,129.38,129.60,130.68,137.88,140.15,141.20,158.44,165.57,167.77,167.88$; MS $(\mathrm{m} / z): 661\left(\mathrm{M}^{+}\right)$; Anal. Calcd for $\mathrm{C}_{39} \mathrm{H}_{40} \mathrm{~N}_{4} \mathrm{O}_{6}: \mathrm{C}, 70.89 ; \mathrm{H}, 6.10 ; \mathrm{N}, 8.48$; Found: $\mathrm{C}, 70.85 ; \mathrm{H}$, $6.15 ; \mathrm{N}, 8.50$.

Diethyl-4-(3-(4-fluorophenyl)-1-phenyl-1H-pyrazol-4-yl)-2,6-dimethyl-1-(4-(3oxomorpholino)phenyl)-1,4-dihydropyridine-3,5-dicarboxylate(3C): Brownsolid; melting range 264-266 ${ }^{\circ}$; IR (KBr):3740, 3601, 3393, 3052, 2936, 2868, 1921, 1734, 1691, 1559, 1525, 1450 , 1290, 1180, 1075, 998, 890, 798, 645, $560 \mathrm{~cm}^{-1}$; MS $(\mathrm{m} / z): 665\left(\mathrm{M}^{+}\right)$; Anal. Calcd for $\mathrm{C}_{38} \mathrm{H}_{37} \mathrm{FN}_{4} \mathrm{O}_{6}$ : C, 68.66; H, 5.61; N, 8.43; Found: C, 68.70; H, 5.55; N, 8.40.

Diethyl-4-(3-(4-chlorophenyl)-1-phenyl-1H-pyrazol-4-yl)-2,6-dimethyl-1-(4-(3oxomorpholino)phenyl)-1,4-dihydropyridine-3,5-dicarboxylate(3D): White solid; melting range $236-238^{\circ} \mathrm{C}$; IR (KBr):3635, 3258, 3110, 3055, 2966, 2870, 2750, 2550, 1790, 1650, 1582, 1533, 1478, 1250, 1190, 1090, 998, 846, 768, 660, $559 \mathrm{~cm}^{-1}$; MS $(\mathrm{m} / z): 680\left(\mathrm{M}^{+}\right)$; Anal. Calcd for $\mathrm{C}_{38} \mathrm{H}_{37} \mathrm{ClN}_{4} \mathrm{O}_{6}: \mathrm{C}, 67.00 ; \mathrm{H}, 5.47 ; \mathrm{N}, 8.23$; Found: C, 67.10; H, 5.50; N, 8.20.

Diethyl-4-(3-(4-bromophenyl)-1-phenyl-1H-pyrazol-4-yl)-2,6-dimethyl-1-(4-(3oxomorpholino)phenyl)-1,4-dihydropyridine-3,5-dicarboxylate(3E): Brownsolid; melting range 238-240 ${ }^{\circ} \mathrm{C}$; IR (KBr): 3740, 3645, 3239, 3098, 2965, 2840, 1779, 1678, 1583, 1548, 1475, 1285 , 1186, 1048, 1037, 837, 719, 689, $550 \mathrm{~cm}^{-1}$; MS $(\mathrm{m} / \mathrm{z}): 725\left(\mathrm{M}^{+}\right)$; Anal. Calcd for $\mathrm{C}_{38} \mathrm{H}_{37} \mathrm{BrN}_{4} \mathrm{O}_{6}: \mathrm{C}$, 62.90; H, 5.14; N, 7.72; Found: C, 62.95; H, 5.10; N, 7.68.

Diethyl-4-(3-(3-bromophenyl)-1-phenyl-1 H-pyrazol-4-yl)-2,6-dimethyl-1-(4-(3oxomorpholino)phenyl)-1,4-dihydropyridine-3,5-dicarboxylate(3F): Brownish solid; melting range $236-240^{\circ} \mathrm{C}$; IR (KBr): $3389,3289,3089,2979,2880,2755,2550,1658,1620,1587,1418$, 1349, 1242, 1190, 1077, 998, 885, 788, 684, 655, 588, $515 \mathrm{~cm}^{-1}$; MS $(\mathrm{m} / \mathrm{z}): 725\left(\mathrm{M}^{+}\right)$; Anal. Calcd for $\mathrm{C}_{38} \mathrm{H}_{37} \mathrm{BrN}_{4} \mathrm{O}_{6}$ : C, 62.90; H, 5.14; N, 7.72; Found: C, 62.87; H, 5.12; N, 7.65.

Diethyl-4-(3-(2-methoxyphenyl)-1-phenyl-1H-pyrazol-4-yl)-2,6-dimethyl-1-(4-(3oxomorpholino)phenyl)-1,4-dihydropyridine-3,5-dicarboxylate(3G): Brown solid; melting range $220-225^{\circ} \mathrm{C}$; $\operatorname{IR}(\mathrm{KBr}): 3640,3232,3070,2969,2871,1794,1659,1590,1531,1415,1260$, 1130, 1028, 878, 734, 660, $555 \mathrm{~cm}^{-1}$; MS $(\mathrm{m} / z): 677\left(\mathrm{M}^{+}\right)$; Anal. Calcd for $\mathrm{C}_{39} \mathrm{H}_{40} \mathrm{~N}_{4} \mathrm{O}_{7}: \mathrm{C}, 69.21$; H, 5.96; N, 8.28; Found: C, 69.25; H, 5.99; N, 8.32.

Diethyl-4-(3-(4-methoxyphenyl)-1-phenyl-1 H-pyrazol-4-yl)-2,6-dimethyl-1-(4-(3oxomorpholino)phenyl)-1,4-dihydropyridine-3,5-dicarboxylate(3H): Brown solid; melting range $226-230^{\circ} \mathrm{C}$; IR (KBr): 3620, 3240, 3114, 3058, 2933, 2870, 1750, 1624, 1590, 1331, 1261, 1205, 1170, 1038, 828, 794, 655, $560 \mathrm{~cm}^{-1}$; MS $(\mathrm{m} / \mathrm{z}): 677\left(\mathrm{M}^{+}\right)$; Anal. Calcd for $\mathrm{C}_{39} \mathrm{H}_{40} \mathrm{~N}_{4} \mathrm{O}_{7}: \mathrm{C}$, 69.21; H, 5.96; N, 8.28; Found: C, 69.18; H, 5.94; N, 8.30.

Diethyl-2,6-dimethyl-4-(3-(3-nitrophenyl)-1-phenyl-1 $H$-pyrazol-4-yl)-1-(4-(3oxomorpholino)phenyl)-1,4-dihydropyridine-3,5-dicarboxylate(3I): Brown solid; melting range 250-252 ${ }^{\circ} \mathrm{C}$; IR (KBr):3650, 3537, 3238, 3098, 2968, 2878, 1770, 1665, 1588, 1530, 1483, 1366, 1327, 1243, 1199, 1071, 1025, 998, 844, 730, 688, 548 $\mathrm{cm}^{-1}$; MS $(\mathrm{m} / \mathrm{z}): 692\left(\mathrm{M}^{+}\right)$; Anal. Calcd for $\mathrm{C}_{38} \mathrm{H}_{37} \mathrm{~N}_{5} \mathrm{O}_{8}$ : C, 65.98; H, 5.39; N, 10.12; Found: C, 66.10; H, 5.42; N, 10.10.

Diethyl-2,6-dimethyl-4-(3-(4-nitrophenyl)-1-phenyl-1H-pyrazol-4-yl)-1-(4-(3-

oxomorpholino)phenyl)-1,4-dihydropyridine-3,5-dicarboxylate(3J): Brown solid; melting range $250-255^{\circ} \mathrm{C}$; IR (KBr):3660, 3238, 3090, 2943, 2881, 1760, 1640, 1580, 1545, 1480, 1266, 1170 , 1072, 1035, 800, 880, 738, 667, 589 $\mathrm{cm}^{-1}$; MS $(\mathrm{m} / \mathrm{z})$ : $692\left(\mathrm{M}^{+}\right)$; Anal. Calcd for $\mathrm{C}_{38} \mathrm{H}_{37} \mathrm{~N}_{5} \mathrm{O}_{8}: \mathrm{C}$, 65.98; H, 5.39; N, 10.12; Found: C, 65.94; H, 5.40; N, 10.14 .

Dimethyl-4-(1,3-diphenyl-1H-pyrazol-4-yl)-2,6-dimethyl-1-(4-(3-oxomorpholino)phenyl)-1,4dihydropyridine-3,5-dicarboxylate(3K): Brownsolid; melting range $252-255^{\circ} \mathrm{C}$; $\mathrm{IR}(\mathrm{KBr}): 3240$, 3113, 2968, 1649, 1591, 1521, 1411, 1346, 1261, 1201, 1128, 1028, 904, 835, 790, 732, 659, 555, 453, $426 \mathrm{~cm}^{-1}$; ${ }^{\mathrm{H}}$ NMR: $\delta$ 2.239-2.241 (s, 6H,), $3.440(\mathrm{~s}, 6 \mathrm{H}), 3.466-3.490(\mathrm{t}, 2 \mathrm{H}), 3.512-3.535(\mathrm{t}$, $2 \mathrm{H}), 4.312(\mathrm{~s}, 2 \mathrm{H}), 5.432-5.442(\mathrm{~s}, 1 \mathrm{H}), 6.784-7.298(\mathrm{~d}, 4 \mathrm{H}, J=7.0 \mathrm{~Hz}), 7.395-7.449(\mathrm{t}, 2 \mathrm{H}), 7.461-$ $7.482(\mathrm{t}, 1 \mathrm{H}), 7.502-7.521(\mathrm{t}, 1 \mathrm{H}), 7.522-7.556(\mathrm{t}, 2 \mathrm{H}), 7.664-7.693(\mathrm{~d}, 2 \mathrm{H}, J=6.5 \mathrm{~Hz}), 7.704-7.740$ 
(d, 2H), 8.610 (s, 1H); ${ }^{13} \mathrm{C}$ NMR: 14.20, 16.40, 28.18, 42.03, 60.10, 64.34, 66.70, 105.30, 119.45, $124.49,126.50,127.55,128.48,129.59,130.61,137.85,140.05,143.19,160.44,165.60,167.70$; MS $(\mathrm{m} / z): 619\left(\mathrm{M}^{+}\right)$; Anal. Calcd for $\mathrm{C}_{36} \mathrm{H}_{34} \mathrm{~N}_{4} \mathrm{O}_{6}$ : C, 69.89; H, 5.54; N, 9.06; Found: $\mathrm{C}, 69.92 ; \mathrm{H}$, $5.58 ; \mathrm{N}, 9.10$.

Dimethyl-2,6-dimethyl-1-(4-(3-oxomorpholino)phenyl)-4-(1-phenyl-3-(p-tolyl)-1H-pyrazol-4yl)-1,4-dihydropyridine-3,5-dicarboxylate(3L):Brown solid; melting range $236-238^{\circ} \mathrm{C} ; \mathrm{R}_{\mathrm{f}} 0.44$ (3:7 hexane-EtOAc); IR (KBr): 3233, 3188, 3064, 2918, 2864, 1741, 1680, 1624, 1542, 1449, 1390, 1280, 1213, 1136, 1035, 966, 835, 768, 670, $595 \mathrm{~cm}^{-1}$; MS $(\mathrm{m} / z): 633\left(\mathrm{M}^{+}\right)$; Anal. Calcd for $\mathrm{C}_{37} \mathrm{H}_{36} \mathrm{~N}_{4} \mathrm{O}_{6}$ : C, 70.24; H, 5.74; N, 8.86; Found: C, 70.28; H, 5.78; N, 8.83.

Dimethyl-4-(3-(4-fluorophenyl)-1-phenyl-1H-pyrazol-4-yl)-2,6-dimethyl-1-(4-(3oxomorpholino)phenyl)-1,4-dihydropyridine-3,5-dicarboxylate(3M): Off brown solid; melting range $242-245^{\circ} \mathrm{C}$; IR (KBr):3280, 3145, 2836, 2755, 2350, 1740, 1638, 1556, 1522, 1475, 1377, 1285, 1195, 998, 817, 769, 668, $520 \mathrm{~cm}^{-1}$; MS $(\mathrm{m} / \mathrm{z}): 637\left(\mathrm{M}^{+}\right)$; Anal. Calcd for $\mathrm{C}_{36} \mathrm{H}_{33} \mathrm{FN}_{4} \mathrm{O}_{6}$ : C, 67.91; H, 5.22; N, 8.80; Found: C, 67.94; H, 5.20; N, 8.85.

Dimethyl-4-(3-(4-chlorophenyl)-1-phenyl-1H-pyrazol-4-yl)-2,6-dimethyl-1-(4-(3oxomorpholino)phenyl)-1,4-dihydropyridine-3,5-dicarboxylate(3N): Brown solid; melting range $255-258^{\circ} \mathrm{C}$; IR (KBr):3244, 3188, 3063, 2938, 2866, 1755, 1665, 1632, 1438, 1390, 1280, $1228,1145,1055,955,845,780,688,592 \mathrm{~cm}^{-1}$; MS $(\mathrm{m} / \mathrm{z}): 653\left(\mathrm{M}^{+}\right)$; Anal. Calcd for $\mathrm{C}_{36} \mathrm{H}_{33} \mathrm{ClN}_{4} \mathrm{O}_{6}: \mathrm{C}, 66.20 ; \mathrm{H}, 5.09 ; \mathrm{N}, 8.58$; Found: C, 66.18; H, 5.15; N, 8.60.

Dimethyl-4-(3-(4-bromophenyl)-1-phenyl-1H-pyrazol-4-yl)-2,6-dimethyl-1-(4-(3oxomorpholino)phenyl)-1,4-dihydropyridine-3,5-dicarboxylate(3O): Brown solid; melting range $26-266^{\circ} \mathrm{C}$; IR (KBr):3270, 3150, 3059, 2950, 2854, 1650, 1598, 1488, 1337, 1298, 1230 , 1185, 1036, 979, 837, 757, 665, 558 $\mathrm{cm}^{-1}$; MS $(\mathrm{m} / \mathrm{z}): 698\left(\mathrm{M}^{+}\right)$; Anal. Calcd for $\mathrm{C}_{36} \mathrm{H}_{33} \mathrm{BrN}_{4} \mathrm{O}_{6}: \mathrm{C}$, 61.98; H, 4.77; N, 8.03; Found: C, 62.01; H, 4.80; N, 8.10.

Dimethyl-4-(3-(3-bromophenyl)-1-phenyl-1H-pyrazol-4-yl)-2,6-dimethyl-1-(4-(3oxomorpholino)phenyl)-1,4-dihydropyridine-3,5-dicarboxylate(3P): Off brown solid; melting range $250-254^{\circ} \mathrm{C}$; IR (KBr):3330, 3220, 3165, 3073, 2929, 2880, 1670, 1560, 1442, 1360, 1271, 1188, 1078, 969, 830, 750, 678, 523 $\mathrm{cm}^{-1}$; MS $(\mathrm{m} / \mathrm{z})$ : $698\left(\mathrm{M}^{+}\right)$; Anal. Calcd for $\mathrm{C}_{36} \mathrm{H}_{33} \mathrm{BrN}_{4} \mathrm{O}_{6}$ : C, 61.98 ; H, 4.77; N, 8.03; Found: C, 61.92; H, 4.75; N, 8.00.

Dimethyl-4-(3-(2-methoxyphenyl)-1-phenyl-1H-pyrazol-4-yl)-2,6-dimethyl-1-(4-(3oxomorpholino)phenyl)-1,4-dihydropyridine-3,5-dicarboxylate(3Q): Brown solid; melting range $266-268^{\circ} \mathrm{C}$; IR (KBr): 3350, 3260, 3180, 3078, 2950, 2870, 1698, 1599, 1490, 1430, 1270 , 1180, 1075, 1040, 875, 784, 682, $598 \mathrm{~cm}^{-1}$; MS $(\mathrm{m} / \mathrm{z}): 649\left(\mathrm{M}^{+}\right)$; Anal. Calcd for $\mathrm{C}_{37} \mathrm{H}_{36} \mathrm{~N}_{4} \mathrm{O}_{7}: \mathrm{C}$, 68.51 ; H, 5.59; N, 8.64; Found: C, 68.53; H, 5.61; N, 8.60.

Dimethyl-4-(3-(4-methoxyphenyl)-1-phenyl-1H-pyrazol-4-yl)-2,6-dimethyl-1-(4-(3oxomorpholino)phenyl)-1,4-dihydropyridine-3,5-dicarboxylate(3R):Off brown solid; melting range $260-265^{\circ} \mathrm{C}$; IR $(\mathrm{KBr}): 3230,3188,3078,2939,2899,1744,1668,1540,1480,1340,1310$, 1298, 1167, 1073, 880, 770, 677, $594 \mathrm{~cm}^{-1}$; MS $(\mathrm{m} / \mathrm{z}): 649\left(\mathrm{M}^{+}\right)$; Anal. Calcd for $\mathrm{C}_{37} \mathrm{H}_{36} \mathrm{~N}_{4} \mathrm{O}_{7}: \mathrm{C}$, 68.51; H, 5.59; N, 8.64; Found: C, 68.53; H, 5.61; N, 8.68.

Dimethyl-2,6-dimethyl-4-(3-(3-nitrophenyl)-1-phenyl-1H-pyrazol-4-yl)-1-(4-(3oxomorpholino)phenyl)-1,4-dihydropyridine-3,5-dicarboxylate(3S): Brown solid; melting range 244-246 ${ }^{\circ} \mathrm{C}$; IR (KBr):3255, 3180, 3076, 2959, 2870, 1750, 1668, 1533, 1475, 1333, 1310, 1290, 1182, 1078, 884, 775, 677, 597 $\mathrm{cm}^{-1}$; MS $(\mathrm{m} / \mathrm{z}): 664\left(\mathrm{M}^{+}\right)$; Anal. Calcd for $\mathrm{C}_{36} \mathrm{H}_{33} \mathrm{~N}_{5} \mathrm{O}_{8}: \mathrm{C}, 65.15 ; \mathrm{H}$, 5.01 ; N, 10.55; Found: C, 65.25; H, 5.10; N, 10.60 .

Dimethyl-2,6-dimethyl-4-(3-(4-nitrophenyl)-1-phenyl-1H-pyrazol-4-yl)-1-(4-(3-

oxomorpholino)phenyl)-1,4-dihydropyridine-3,5-dicarboxylate(3T): Brown solid; melting range $234-238^{\circ} \mathrm{C}$; IR(KBr):3180, 3077, 2945, 2875, 1715, 1667, 1593, 1559, 1477, 1286, 1159, 1098, 1060, 886, 790, 687, $590 \mathrm{~cm}^{-1}$; MS $(\mathrm{m} / z): 664\left(\mathrm{M}^{+}\right)$; Anal. Calcd for $\mathrm{C}_{36} \mathrm{H}_{33} \mathrm{~N}_{5} \mathrm{O}_{8}: \mathrm{C}, 65.15 ; \mathrm{H}, 5.01$; N, 10.55; Found: C, 65.13; H, 5.03; N, 10.53 . 


\section{Conclusion}

In summary, we have described the synthesis of substituted 1,4-dihydropyridine derivatives in moderate yield. The reaction of various pyrazole aldehydes (PALs)withethylacetoacetate or methylacetoacetate (active methylene group containing compouns) and 4-(4-aminophenyl) morpholin-3-one was afforded the 1,4-dihydropyridine derivatives in good yield.All synthesized compounds were characterized by IR, NMR and Mass spectrometry analysis and are incorporate with the structure of compounds 3A-3T In vitro affectivity of antimicrobial agents can be demonstrated by observing their capacity to inhibit bacterial growth on suitable media. The production of a zone depends on two factors namely bacterial growth and concentration of antimicrobial agent. The hole/well punch method was first used by Bennett. This diffusion method has proved more effective than many other methods. According to Lt. General Raghunath the well technique is 5-6 times more sensitive than using disk method.

\section{ACKNOWLEDGEMENT}

Authors are thankful to Department of Chemistry for providing laboratory facilities and also thankful to National Facility for Drug Discovery (NFDD), Saurashtra Universityfor providing spectral analysis of the compounds.

\section{References}

[1] Imming P.; Graf M.; Tries S.; Hirschelmann R.; Krause E.; Pawlitzki G. Anti-Inflammatory planar chiral [2.2] paracyclophaneacetic acid enantiomers, Inflamm. Res.2001, 50, 371-374.

[2] Agudoawu, S.A.; Yiu, SH,; Wallace, J.L.; Knaus, E.E. Synthesis and analgesic activity of 2methyl-2-[1-(3-benzoyl-4-substituted-1,4-dihydropyridyl)] acetic acid methyl esters, acetic acids and acetamides, Arch. Pharm. (Weinheim) 1999, 332, 213-218.

[3] Shi, K. H.; Lim, S. S.; Jung, S. H.; Ji, J.; Lee, E. B.; Kim, D. C.; Shin, K. J.; Kim, D. J.; Park, S. W. Synthesis and anti-inflammatory and alalgesic activities of pyridyloxy-and phenoxyalkanoic acid derivatives, Arch. Pharm. Res.2000, 23, 17-21.

[4] Gred, D.; Warner, K. Cyclooxygenase inhibitors-current status and future prospects, Eur. J. Med. Chem.2001, 36, 109-126.

[5] Figueiredo, J. M.; Camara, C. A.; Amarante, E. G.; Miranda, A. L. P.; Santos, F. M.; Rodrigues, C. R.; Fraga, C. A. M.; Barreiro, E. J. Design and synthesis of novel potent antinociceptive agents methyllimidazoyl-N-acylhydrazone derivatives, Bioorg. Med. Chem.2000, 8, 2243-2238.

[6] Shinde, D.B.; Shinde, N.D.; Shingare, M.S.; Synthesis of 1,4-dyhydropyridines and their hypotensive activity. Indian J. Chem.1995, 34B, 920-922.

[7] Shah, A.; Gaveriya, H.; Motohashi, N.; Kawase, M.; Saito, S.; Sakagami, H.; SatohTada, Y.; Solmosi, A.; Walfard, K.; Molnar, J. 3,5-Diacetyl-1,4-dihydropyridines synthesis and MDR reversal in tumor cells, Anticancer. Res. 2000, 20, 373-377.

[8] Di Donato, M.; Maioli, M.; Venturi.; Burgisser, C.; Fantini, F.; Biamino, G.; Marchionni, N. Acute hemodynamic effects of intravenous diperdipine, a new dihydropyridine derivative, in coronary heart disease. Am. Heart J. 1991, 121, 776-781. 